\title{
SOLUTION OF LINEAR DIFFERENTIAL EQUATIONS USING OPERATIONAL MATRIX OF BERNOULLI ORTHONORMAL POLYNOMIALS
}

\author{
MITHILESH SINGH, SEEMA SHARMA, AND SUNIL RAWAN ${ }^{\dagger}$
}

\author{
Date of Receiving : $\quad 16.10 .2019$ \\ Date of Revision : $\quad 11.03 .2020$ \\ Date of Acceptance : 12.03 .2020
}

\begin{abstract}
In this paper, a new numerical method has been proposed, which is based on the normalized Bernoulli polynomials for solving linear differential equations. An orthonormal family of polynomials of degree $\mathrm{n}$ from the $n^{\text {th }}$ degree Bernoulli polynomials has been constructed. The properties of Bernoulli polynomials have been employed to construct the operational matrices of integration. These polynomials are orthogonalized, normalized and then their operational matrix of integration is derived. To demonstrate the efficiency and accuracy of the proposed method, the solutions of two numerical examples have been illustrated.
\end{abstract}

\section{Introduction}

A real-life system or phenomenon whether physical, sociological, economic, etc., gives rise to what is known as a mathematical model when described mathematically. Usually these models involve assumptions about the rate of change of one or more variables and can be expressed by means of differential, integral or integro-differential equations, or systems of them $[1,2,3]$. It is often required to employ numerical techniques to obtain an approximate solution. In 2000, He[4] studied variational iteration method to find approximate solutions to autonomous ordinary differential systems. Razzaghi and Yousefi[5] introduced the Legendre wavelets operational matrix of integration to solve problems such as identification, analysis and optimal control in 2001. They also introduced an operational matrix of integration based on sine and cosine wavelets and found its applications in calculus of variations. For details, see[6]. In 2010, Yousefi and Behroozifar[7] formulated B-polynomials operational matrix method. Later in the same year, Saadatmandi and Dehghan[8] used Legendre series together with the Legendre operational matrix of fractional derivatives to obtain numerical integration of fractional

Key words and phrases. Orthonormal Bernoulli polynomials; Operational matrix; Differential equation.

Communicated by: Silvia Seminara

${ }^{\dagger}$ Corresponding author. 